\title{
Atracurium Besylate: Chemo-degradable and bio- degradable pharmaceutically active drug
}

\author{
Ahmed Ma'mun ${ }^{1 *}$, Mohamed K Abd El-Rahman ${ }^{1}$ and Mohamed Abd El-Kawy ${ }^{2}$ \\ ${ }^{1}$ Analytical Chemistry Department, Faculty of Pharmacy, Cairo University, Kasr-El Aini Street, Cairo, 11562, Egypt \\ ${ }^{2}$ Pharmaceutical Chemistry Department, Faculty of Pharmaceutical Sciences \& Pharmaceutical Industries, Future University, 12311, Cairo, Egypt
}

In recent years, the whole field of ion-selective electrodes (ISEs) in pharmaceutical sciences has expanded far beyond its original roots. The diverse range of opportunities offered by ISEs was broadly used in a number of pharmaceutical applications, with topics presented ranging from bioanalysis of drugs and metabolites, to protein binding studies, green analytical chemistry, impurity profiling, and drug dissolution in biorelevant media. Inspired from these advances and with the aim of extending the functional capabilities of ISEs, the primary focus of the present paper is the utilization of ISE as a tool in personalized medicine. Given the opportunity to explore biological events in real-time (such as drug metabolism) could be central to personalized medicine. (ATR) is a chemo-degradable and bio-degradable pharmaceutically active drug (Figure 1). Laudanosine (LDS) is the major degradation product and metabolite of ATR and is potentially toxic and reported to possess epileptogenic activity which increases the risk of convulsive effects. In this work, ATR have been subjected to both chemical and biological hydrolysis, and the course of the reactions is monitored by means of an ISE. In this study, we have designed an efficient real-time tracking strategy which substantially resolve the challenges of the ATR chemical and biological degradation kinetics. By utilizing a potentiometric sensor, tracking of ATR chemical and biological degradation kinetics can be performed in a very short time with excellent accuracy.

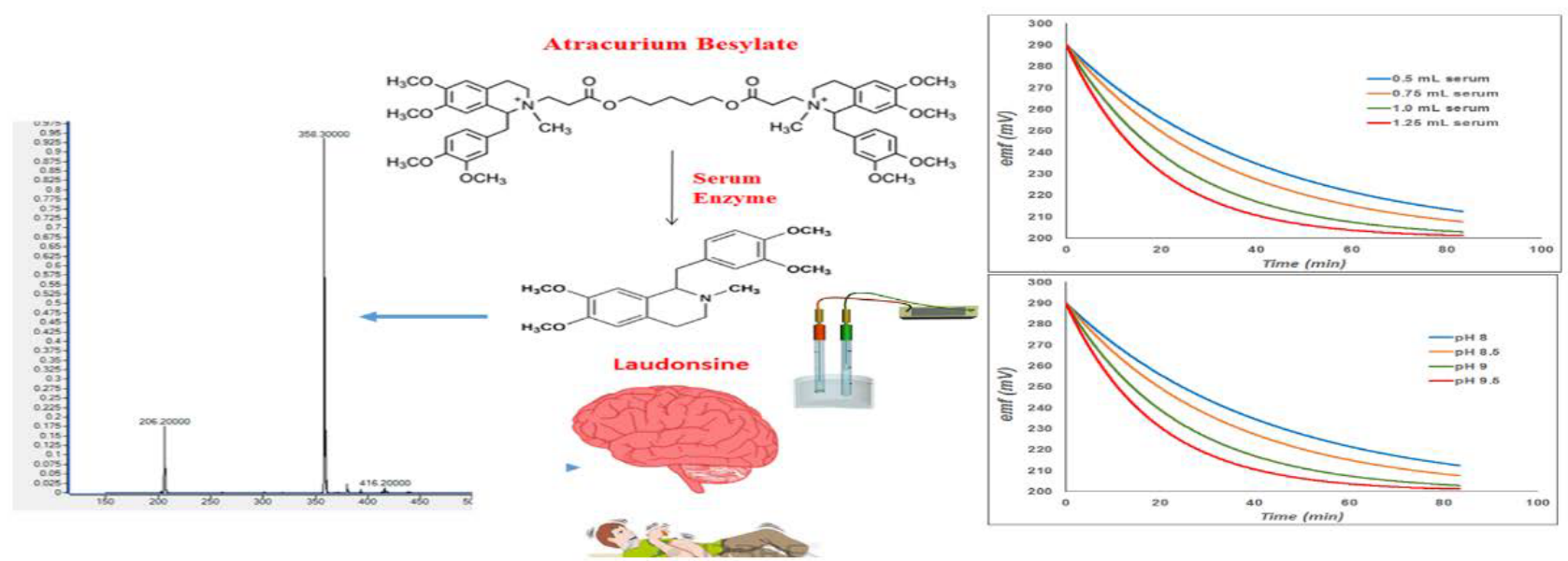

Figure 1. Pointing to the importance of monitoring the plasma level of ATR and Laudanosine

Copyright: (C2018 Ma'mun A. This is an open-access article distributed under the terms of the Creative Commons Attribution License, which permits unrestricted use, distribution, and reproduction in any medium, provided the original author and source are credited.
${ }^{*}$ Correspondence to: Ma'mun A, Analytical Chemistry Department, Faculty of Pharmacy, Cairo University, Kasr-El Aini Street, Cairo, 11562, Egypt, E-mail: ahmed.mamun@orchidiapharmaceutical.com

Received: July 24, 2018; Accepted: August 06, 2018; Published: August 09, 2018 